\title{
Equilibrium Analysis and Social Optimization in Cognitive Radio Networks
}

\author{
Zhen Zeng ${ }^{1,2}$ and Gang Wang ${ }^{1, *}$ \\ ${ }^{1}$ Communication Research Center, Harbin Institute of Technology, \\ Harbin, 150001, China \\ ${ }^{2}$ Harbin University of Science and Technology, \\ Harbin, 150080, China \\ zengzhen8mei@126.com
}

\begin{abstract}
This paper investigates the admission control problem in cognitive radio networks (CRNs) from the economic viewpoint. Each secondary user (SU) has the option of accessing the spectrum or balking on account of the trade-off between the service profit and the delay cost. In order to avoid disturbing the transmission of primary users (PUs), the secondary user requires detecting the presence of PUs before starting its own communication. In addition, whenever a PU appears on the working channel, the SU must halt its transmission to guarantee the communication quality of PU. Therefore, regarding the actions of $P U$ and $S U$, the licensed channel can be seen as a server switching between two working modes ON/OFF and each SU's data packet, call or connection as a customer. We propose a partially observable M/M/I queueing model to describe the decision process of SUs. Based on the queueing model, we present two admission control strategies to maximize the welfare of individual SU and the whole society respectively.
\end{abstract}

Keywords: Cognitive radio, game theory, equilibrium strategy, social optimization strategy

\section{Introduction}

The literature on spectrum access issue applying queueing theory in cognitive radio networks is rich. However, most of them focus on the spectrum selection methods [1], [2]. Very few works $[3,4]$ consider the equilibrium behavior and discuss the spectrum access mechanism to control the number of SUs from the economic standpoint in cognitive radio networks. In the system, each arriving SU has the option of accessing the spectrum or balking based on the trade-off between the benefits associated with service and the costs associated with waiting [5]. Since each individual SU's decision is capable of exerting influence on other SUs, the procedure can be modeled as a noncooperative game. The equilibrium strategy and socially optimal strategy are the central issues in the model. In [3], Li et al., assumed that SUs could derive the information on the number of waiting SUs in the system and then decide whether to queue based on the information. In contrast, in [4], Do et al., discussed the decision process of SUs in which no information could be obtained prior to SUs' actions. The two papers both analyzed SU's individual behavior in equilibrium and proposed optimal strategies to maximize the social welfare. However, in a real scenario, SUs can observe the channel state by spectrum sensing but seldom get the queue length information. Therefore, we adopt the partially observable queue model to describe the decision process of SUs in this paper, which is more general and practical. 
Similar to [4], we apply the queueing theory with server breakdowns to study the spectrum access control. The licensed channel, temporarily used by SUs, can be treated as a server and each SU's data packet, call or connection as a customer. To ensure PU's service quality, SU is unable to continue its transmission whenever PU reappears on the operating channel [6]. Therefore, we view the arrival of PU as a server breakdown. Upon arriving, SUs can use the sensing ability to learn about the current channel state and then decide whether to join or balk based on the sensing information.

The major contributions of this paper include: We model the cognitive radio system as a partially observable M/M/1 queue with server-breakdown. Based on the model, we investigate the individual equilibrium strategy and social optimization strategy in cognitive radio networks.

\section{System Model}

We focus on a single channel cognitive radio system with multiple SUs. Regarding PU's activities, the licensed channel can be seen as a server which switches between two working modes ON/OFF, where ON state implies that PU is absent and OFF state implies the channel is utilized by PU. We assume the holding periods in ON and OFF states are exponentially distributed with rates $\theta$ and $\eta$, respectively. The SUs arrive according to a Poisson process of rate $\lambda$ and service times follow independent exponential distribution with rate $\mu$. In addition, the SU service discipline is Firstcome First-served (FCFS).

We use a pair $(N(t), I(t))$ to describe the state of the system at time $t$, where $N(t)$ denotes the queue-size which includes the SU in service and $I(t)$ denotes the current state of the licensed channel (0: ON, 1: OFF). Obviously, the process $\{(N(t), I(t))$ : $\mathrm{t}$ $\geq 0\}$ is a continuous time Markov chain with non-zero transition rates. To model the decision process, we suppose the reward for a SU to complete transmission is $R$ units and the cost caused by waiting is $C$ units per time unit. The welfare of each SU consists of benefits associated with service, from which waiting costs are subtracted; that is: $R$ $C T$. Every individual SU wants to maximize its expected net benefit. Furthermore, we assume that the SUs which resolve to access the channel are forbidden to renege.

\section{Equilibrium Analysis and Social Optimization}

In this study, we assume that an arriving SU can derive the information on the channel's current state prior to its action. A vector $\left(q_{0}, q_{1}\right)$ is used to specify the mixed strategy of SUs, where $q_{i}$ denotes the joining probability when SUs find the channel in state $i(i=0,1)$. If all SUs follow the same mixed strategy $\left(q_{0}, q_{1}\right)$, then the system follows a Markov chain with state space $\Omega=\{(n, i) \mid \mathrm{n} \geq 0, i=0,1\}$. Therefore, when the channel is in state $i$, the effective arrival rate equals $\lambda_{i}=\lambda q_{i}$. Figure 1 shows the state transition rate diagram.

Because the holding periods of the channel in ON and OFF states are exponentially distributed with rates $\theta$ and $\eta$ respectively, the probability of the channel staying in ON state is $P_{0}=\eta /(\theta+\eta)$ and in OFF state is $P_{1}=\theta /(\theta+\eta)$. We consider the stable system in this paper, which means $\mu \eta>\lambda(\theta+\eta)$. Based on above condition, the 


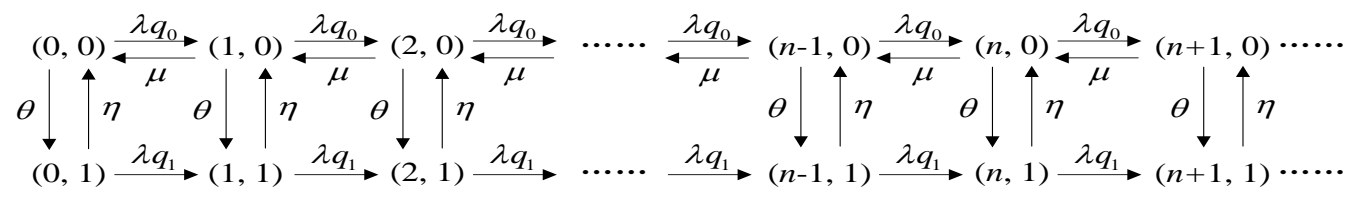

Figure 1. State Transition Rate Diagram for the $\left(q_{0}, q_{1}\right)$ Mixed Strategy

corresponding stationary distribution $\{p(n, i):(n, i) \in \Omega\}$ is obtained by using the following balance equations:

$$
\begin{gathered}
\left(\lambda_{1}+\eta\right) p(0,1)=\theta p(0,0) \\
\left(\lambda_{1}+\eta\right) p(n, 1)=\theta p(n, 0)+\lambda_{1} p(n-1,1), \quad n=1,2, \ldots
\end{gathered}
$$

Now by applying the vertical cuts in Figure 1,

$$
\lambda_{0} p(n, 0)+\lambda_{1} p(n, 1)=\mu p(n+1,0), \quad n=0,1, \ldots
$$

Let $G_{i}(z)$ be the probability generating function (PGF) of the number of SUs in the system when the channel is in state $i$. Therefore, we have

$$
G_{i}(z)=\sum_{n=0}^{\infty} z^{n} p(n, i), \quad i=0,1
$$

Multiplying (2) by $z^{n}$ and summing over $n$, the equation becomes

$$
\left(\lambda_{1}+\eta\right)\left[G_{1}(z)-p(0,1)\right]=\theta\left[G_{0}(z)-p(0,0)\right]+\lambda_{1} z G_{1}(z) .
$$

Similarly, from (3) we have

$$
\lambda_{0} G_{0}(z)+\lambda_{1} G_{1}(z)=\frac{\mu}{z}\left[G_{0}(z)-p(0,0)\right] .
$$

Using (1), (5) and (6), we obtain

$$
G_{0}(z)=\frac{\mu\left(\lambda_{1}+\eta-\lambda_{1} z\right) p(0,0)}{H(z)},
$$

and

$$
G_{1}(z)=\frac{\mu \theta p(0,0)}{H(z)},
$$

where $H(z)$ is given by

$$
H(z)=\lambda_{0} \lambda_{1} z^{2}-\left(\lambda_{0} \lambda_{1}+\lambda_{0} \eta+\lambda_{1} \theta+\lambda_{1} \mu\right) z+\mu\left(\lambda_{1}+\eta\right) .
$$

Summing (3) over $n$ gives

$$
p(0,0)=\frac{\left(\mu-\lambda_{0}+\lambda_{1}\right) P_{0}-\lambda_{1}}{\mu}=\frac{\mu \eta-\lambda_{0} \eta-\lambda_{1} \theta}{\mu(\theta+\eta)} .
$$


From (7)-(10), the mean number $E\left[N_{i}\right]$ of SUs in the system in state $i$ is given by

$$
E\left[N_{0}\right]=\left.\frac{d G_{0}(z)}{d z}\right|_{z=1}=\frac{\lambda_{0} \eta^{2}+\lambda_{1}^{2} \theta+\lambda_{1} \theta \eta}{(\theta+\eta)\left(\mu \eta-\lambda_{0} \eta-\lambda_{1} \theta\right)},
$$

and

$$
E\left[N_{1}\right]=\left.\frac{d G_{1}(z)}{d z}\right|_{z=1}=\frac{\theta\left(\lambda_{0} \eta+\lambda_{1} \theta+\lambda_{1} \mu-\lambda_{0} \lambda_{1}\right)}{(\theta+\eta)\left(\mu \eta-\lambda_{0} \eta-\lambda_{1} \theta\right)} .
$$

According to the closed-form expressions of the conditional sojourn time $T_{i}\left(q_{0}, q_{1}\right)$ of the customers derived in [7], we have the following equations:

$$
T_{0}\left(q_{0}, q_{1}\right)=\frac{\theta}{\mu \eta}+\frac{\mu \eta^{2}+\left(\lambda q_{1}\right)^{2} \theta}{\mu \eta\left(\mu \eta-\lambda q_{0} \eta-\lambda q_{1} \theta\right)},
$$

and

$$
T_{1}\left(q_{0}, q_{1}\right)=\frac{1}{\mu}+\frac{1}{\eta}+\frac{\theta \mu \eta-\lambda^{2} q_{0} q_{1} \theta+\lambda q_{1} \mu \theta}{\mu \eta\left(\mu \eta-\lambda q_{0} \eta-\lambda q_{1} \theta\right)} .
$$

\subsection{Individual Equilibrium Strategy}

The net benefit for a SU who joins the queue is the value of service minus the waiting cost. Therefore, the expected benefit of a SU who finds the channel in state $i$ upon arrival can be expressed as

$$
U_{i}\left(q_{0}, q_{1}\right)=R-C T_{i}\left(q_{0}, q_{1}\right) .
$$

A SU then enters if this value $U_{i}$ is nonnegative. Otherwise, the SU balks. We note that $U_{i}$ is strictly decreasing in $q_{i}$.

Next, we discuss the individual equilibrium strategy of each SU. For excluding a trivial case, we make the following assumption: $U_{0}(0,0)>0$, that is, $R>C(\theta+\eta) / \mu \eta$.Then, we study eight possible ranges of $R$ and discuss the existence of a symmetric Nash equilibrium $\left(q_{0}^{e}, q_{1}^{e}\right)$ in each case.

Case 1: $U_{1}(0,1)<0<U_{1}(0,0)$. That is $C\left(\frac{\mu+\eta+\theta}{\mu \eta}\right)<R<C\left(\frac{1}{\mu}+\frac{\mu+\theta}{\mu \eta-\lambda \theta}\right)$. Due to the continuity and monotonicity of $U_{1}$, there exists a unique $q_{1}^{e}$ satisfying $U_{1}\left(0, q_{1}^{e}\right)=0$ which is given by

$$
q_{1}^{e}=x_{1}=\frac{\mu \eta}{\lambda \theta}-\frac{C \mu(\mu+\theta)}{\lambda \theta(R \mu-C)} .
$$

Furthermore, the expected benefit $U_{0}\left(0, x_{1}\right)<0$. Therefore, we obtain the equilibrium strategy $\left(q_{0}^{e}, q_{1}^{e}\right)=\left(0, x_{1}\right)$ if $y_{1}<x_{1}<1$ where $y_{1}=\frac{\theta(\theta-\eta)+\sqrt{\theta^{2}(\theta-\eta)^{2}+4 \mu^{2} \theta \eta}}{2 \lambda \theta}$. Under this situation, a part $x_{1}$ of SUs that find PU present upon arrival access the channel, while all the arriving SUs who find PU absent decide to balk. 
Case 2: $U_{0}(0,1)<0 \leq U_{1}(0,1)$. That is $C\left(\frac{1}{\mu}+\frac{\mu+\theta}{\mu \eta-\lambda \theta}\right) \leq R<C\left[\frac{\theta}{\mu \eta}+\frac{\mu \eta^{2}+\lambda^{2} \theta}{\mu \eta(\mu \eta-\lambda \theta)}\right]$ if $\lambda\left(\theta \eta+\lambda \theta-\theta^{2}\right)>\mu^{2} \eta$. In this case, even if all arriving SUs access the channel when it is occupied by PU, they all enjoy a non-negative benefit. And even if no other SU joins, the net benefit of a SU who joins is negative when the PU is absent. Therefore, the equilibrium strategy is $\left(q_{0}^{e}, q_{1}^{e}\right)=(0,1)$ which means all SUs access the channel if it is occupied, while no SU access the channel if it is available.

Case 3: $U_{0}(1,0)<0<U_{0}(0,0)$. That is $C\left(\frac{\theta+\eta}{\mu \eta}\right)<R<C\left(\frac{\theta}{\mu \eta}+\frac{1}{\mu-\lambda}\right)$. Due to the continuity and monotonicity of $U_{0}$, there exists a unique $q_{0}^{e}$ satisfying $U_{0}\left(q_{0}^{e}, 0\right)=0$ which is given by

$$
q_{0}^{e}=x_{2}=\frac{\mu}{\lambda}-\frac{C \mu \eta}{\lambda(R \mu \eta-C \theta)} .
$$

Furthermore, the expected benefit $U_{1}$ must satisfy $U_{1}\left(x_{2}, 0\right)<0$. Therefore, we obtain the equilibrium strategy $\left(q_{0}^{e}, q_{1}^{e}\right)=\left(x_{2}, 0\right)$ if $y_{2}<\mu^{2}$ where $y_{2}=\lambda x_{2}(\mu+\eta-\theta)$. Under this situation, a part $x_{2}$ of SUs who find PU absent upon arrival access the channel, while all the arriving SUs who find PU present decide to balk.

Case 4: $U_{0}(1,1)<0<U_{0}(0,0)$ and $U_{1}(1,1)<0<U_{1}(0,0)$. That is $C\left(\frac{\mu+\theta+\eta}{\mu \eta}\right)<R<\mathrm{min}$ $\left\{C\left[\frac{\mu \eta^{2}+\lambda^{2} \theta}{\mu \eta(\mu \eta-\lambda \eta-\lambda \theta)}+\frac{\theta}{\mu \eta}\right], C\left[\frac{\mu+\eta}{\mu \eta}+\frac{\theta \mu \eta-\lambda^{2} \theta+\lambda \mu \theta}{\mu \eta(\mu \eta-\lambda \eta-\lambda \theta)}\right]\right\}$. There exists a pair $\left(q_{0}^{e}, q_{1}^{e}\right)$ satisfying $U_{0}\left(q_{0}^{e}, q_{1}^{e}\right)=0$ and $U_{1}\left(q_{0}^{e}, q_{1}^{e}\right)=0$ which are given by

$$
q_{0}^{e}=x_{3}=\frac{\mu \eta^{2}(R \eta-C)(R \mu-2 C)+C^{2} \eta\left(\mu^{2}+\eta^{2}\right)}{C \lambda \theta(C \theta-R \mu \eta)}+\frac{C \mu \eta+R \mu \eta \theta-C \theta \eta}{C \lambda \theta},
$$

and

$$
q_{1}^{e}=x_{4}=\frac{C \theta^{2}-C \mu \eta-R \mu \theta \eta-C \eta^{2}+R \mu \eta^{2}}{C \lambda \theta} .
$$

Therefore, we obtain the equilibrium strategy $\left(q_{0}^{e}, q_{1}^{e}\right)=\left(x_{3}, x_{4}\right)$ if $0<x_{3}<1$ and $0<x_{4}<1$. Under this situation, a part $x_{3}$ of SUs join the licensed channel if available, and a part $x_{4}$ of SUs select to join if it is occupied.

Case 5: $U_{0}(1,1)<0<U_{0}(0,1)$. That is $C\left[\frac{\mu \eta^{2}+\lambda^{2} \theta}{\mu \eta(\mu \eta-\lambda \theta)}+\frac{\theta}{\mu \eta}\right]<R<C\left[\frac{\mu \eta^{2}+\lambda^{2} \theta}{\mu \eta(\mu \eta-\lambda \theta-\lambda \eta)}\right.$ $\left.+\frac{\theta}{\mu \eta}\right]$. Therefore, there exists a unique $q_{0}^{e}$ satisfying $U_{0}\left(q_{0}^{e}, 1\right)=0$ which is given by

$$
q_{0}^{e}=x_{5}=\frac{\mu}{\lambda}-\frac{\theta}{\eta}-\frac{C\left(\mu \eta^{2}+\lambda^{2} \theta\right)}{\lambda \eta(R \mu \eta-C \theta)} .
$$

Furthermore, the expected benefit $U_{1}$ must satisfy $U_{1}\left(x_{5}, 1\right) \geq 0$. Therefore, we obtain the 
equilibrium strategy $\left(q_{0}^{e}, q_{1}^{e}\right)=\left(x_{5}, 1\right)$ if $y_{3} \geq \lambda \theta^{2}-\lambda \theta \eta+\mu^{2} \eta-\lambda^{2} \theta$ where $y_{3}=\lambda x_{5}(\mu \eta+$ $\left.\eta^{2}+\lambda \theta-\theta \eta\right)$. Under this situation, a part $x_{5}$ of SUs who find PU absent upon arrival access the channel, while all the arriving SUs who find PU present still decide to join.

Case 6: $U_{1}(1,0)<0 \leq U_{0}(1,0)$. That is $C\left(\frac{\theta}{\mu \eta}+\frac{1}{\mu-\lambda}\right) \leq R<C\left(\frac{\mu+\eta}{\mu \eta}+\frac{\theta}{\mu \eta-\lambda \eta}\right)$ if $\lambda(\mu$

$+\eta-\theta)<\mu^{2}$. In this case, even if all arriving SUs access the channel, they all enjoy a nonnegative benefit when the PU is absent. And even if no other SU joins, the net benefit of a SU who joins is negative when the channel is occupied by PU. Therefore, the equilibrium strategy is $\left(q_{0}^{e}, q_{1}^{e}\right)=(1,0)$.

Case 7: $U_{1}(1,1)<0<U_{1}(1,0)$. That is $C\left(\frac{\mu+\eta}{\mu \eta}+\frac{\theta}{\mu \eta-\lambda \eta}\right)<R<C\left[\frac{\theta \mu \eta-\lambda^{2} \theta+\lambda \mu \theta}{\mu \eta(\mu \eta-\lambda \eta-\lambda \theta)}\right.$

$\left.+\frac{\mu+\eta}{\mu \eta}\right]$. There exists a unique $q_{1}^{e}$ satisfying $U_{1}\left(1, q_{1}^{e}\right)=0$ which is given by

$$
q_{1}^{e}=x_{6}=\frac{(R \mu \eta-C \eta-C \mu)(\mu-\lambda) \eta-C \theta \mu \eta}{\lambda \theta(R \mu \eta-C \eta-C \lambda)} .
$$

Furthermore, the expected benefit $U_{0}\left(1, x_{6}\right) \geq 0$. Therefore, we obtain the equilibrium strategy $\left(q_{0}^{e}, q_{1}^{e}\right)=\left(1, x_{6}\right)$ if $y_{4} \geq \eta-\theta$ where $y_{4}=\frac{\mu^{2} \eta-\lambda^{2} \theta x_{1}^{2}-\lambda^{2} \theta x_{1}-\lambda \mu \eta}{\lambda \eta+\lambda \theta x_{1}}$. Under this situation, a part $x_{6}$ of SUs who find PU present upon arrival access the channel, while all the arriving SUs who find PU absent decide to join.

Case 8: $U_{0}(1,1) \geq 0$ and $U_{1}(1,1) \geq 0$. That is $R \geq \max \left\{C\left[\frac{\theta}{\mu \eta}+\frac{\mu \eta^{2}+\lambda^{2} \theta}{\mu \eta(\mu \eta-\lambda \eta-\lambda \theta)}\right]\right.$,

$\left.C\left[\frac{\mu+\eta}{\mu \eta}+\frac{\theta \mu \eta-\lambda^{2} \theta+\lambda \mu \theta}{\mu \eta(\mu \eta-\lambda \eta-\lambda \theta)}\right]\right\}$. In this case, the equilibrium strategy is $\left(q_{0}^{e}, q_{1}^{e}\right)=(1,1)$ which means the arriving SUs always select to join whatever state the spectrum is in.

We may tend to believe that SUs prefer to access the channel when PU is absent since they do not have to wait for PU's service time. However, by comparing the value of $q_{0}^{e}$ and its corresponding $q_{1}^{e}$, we note that it is just true under Case 3,6 and 7. Therefore, the information that the channel is occupied by PU does not hinder SUs from joining the spectrum. Furthermore, the welfare of a SU obtained from accessing the spectrum decreases with the joining probability of other SUs regardless of the information on the channel state. This shows the avoid-the-crowd (ATC) behavior of SUs, and consequently, at most one equilibrium exists.

\subsection{Socially Optimal Strategy}

The social benefit per time unit when every SU adopts the mixed strategy $\left(q_{0}, q_{1}\right)$ can be expressed as

$$
S_{o}\left(q_{0}, q_{1}\right)=\frac{\lambda \eta q_{0}}{\theta+\eta}\left[R-\frac{C \theta}{\mu \eta}-\frac{C\left(\mu \eta^{2}+\lambda^{2} q_{1}^{2} \theta\right)}{\mu \eta\left(\mu \eta-\lambda q_{0} \eta-\lambda q_{1} \theta\right)}\right]+\frac{\lambda \theta q_{1}}{\theta+\eta}\left[R-\frac{C \theta\left(\mu \eta-\lambda^{2} q_{0} q_{1}+\lambda q_{1} \mu\right)}{\mu \eta\left(\mu \eta-\lambda q_{0} \eta-\lambda q_{1} \theta\right)}\right.
$$




$$
\left.-\frac{C(\mu+\eta)}{\mu \eta}\right] .
$$

The goal of social optimization is to find a strategy $\left(q_{0}^{e}, q_{1}^{e}\right)$ to maximize the net benefit $S_{o}$. Considering that $\left(q_{0}, q_{1}\right) \in M=[0,1] \times[0,1]$, we discuss the optimization problem in five subregions: $M_{1}=\{0\} \times[0,1], M_{2}=\{1\} \times[0,1], M_{3}=(0,1) \times(0,1), M_{4}=[0,1] \times\{0\} \quad$ and $M_{5}=[0,1] \times\{1\}$. Therefore, we can obtain the socially optimal strategy $\left(q_{0}^{s}, q_{1}^{s}\right)$ corresponding to the maximum value of $S_{o}$ among these extreme points in five subregions. If the socially optimal joining probability $q_{i}^{s}$ is less than the equilibrium one $q_{i}^{e}$, an admission fee should be imposed to discourage SUs from accessing the channel; otherwise, an allowance should be adopted to attract more SUs to join.

\section{Numerical Results}

In this section, we present some numerical results to illustrate the equilibrium and social optimization strategies. Here we focus on the joining probabilities of SUs and the corresponding social benefit by adopting the two different access strategies.

(a)

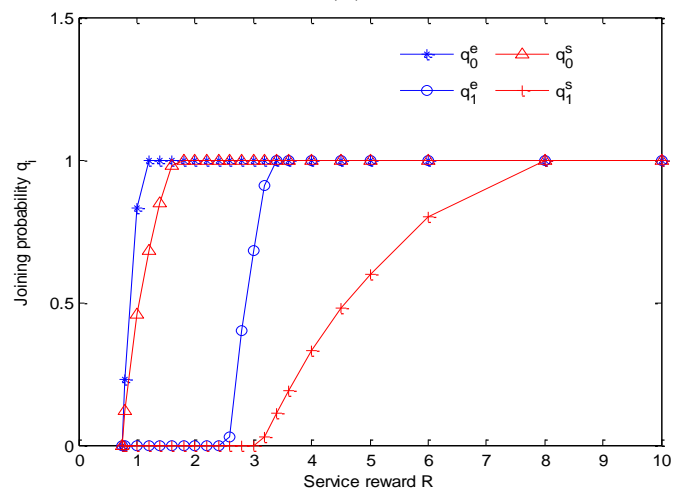

(b)

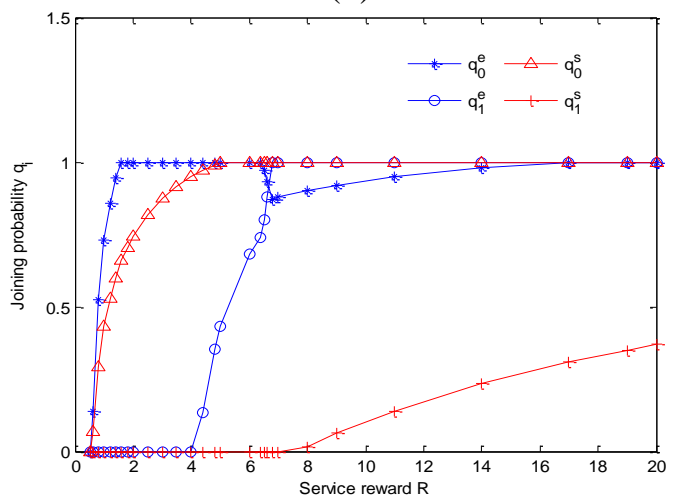

Figure 2. Equilibrium and Socially Optimization Joining Probabilities of SUs with Respect to R, a for $\lambda=0.8, \mu=2, \theta=0.3, \eta=0.6, C=1$; b for $\lambda=1.7, \mu=2.4$, $\theta=0.1, \eta=0.3, C=1$

Figure 2 shows the equilibrium and social optimization joining probabilities with respect to the service reward $R$ under different system parameters. The equilibrium and social optimization access probabilities all go towards one as $R$ increases. In other words, joining is always the best choice both from the viewpoint of individual SU and the overall SUs when $R$ is large enough, irrespectively of the licensed channel's state. We observe that the social optimization joining probability $q_{i}^{s}$ is growing with $R$, which is intuitive. By comparing Figure 2(a) and Figure 2(b), we notice the equilibrium probability $q_{1}^{e}$ is a monotone nondecreasing function of $R$ while $q_{0}^{e}$ acts as a non-monotone function of $R$. This fact demonstrates that the individual SU's optimal access strategy depends on the relation between $R$ and the other system parameters. Moreover, it is worth specifying that the equilibrium probability $q_{i}^{e}$ is greater than or equal to the social optimization probability $q_{i}^{s}$ in Figure 2(a). In this case, an admission fee is needed to motivate SUs to adopt the joining 
probability $q_{i}^{s}$ rather than $q_{i}^{e}$. In contrast, the relative ordering of $q_{0}^{e}$ and $q_{0}^{s}$ are different in Figure 2(b) where we can see $q_{0}^{e}$ is less than $q_{0}^{s}$ in some region. Under this situation, an allowance is required to encourage more SUs to access the free channel.

The behavior of social benefit as $R$ varies is depicted in Figure 3 and the same system parameters are set in Figure 3 as well as those in Figure 2. Note that the optimal social benefit $S_{o}$ is strictly increasing with the rise in $R$ and the equilibrium social benefit $S_{e}$ is not monotonic. In the meanwhile, $S_{o}$ is always greater than or equal to $S_{e}$ as expected. The difference of values between equilibrium and optimal social benefit is due to the harmful impact which SUs impose on future SUs.

(a)

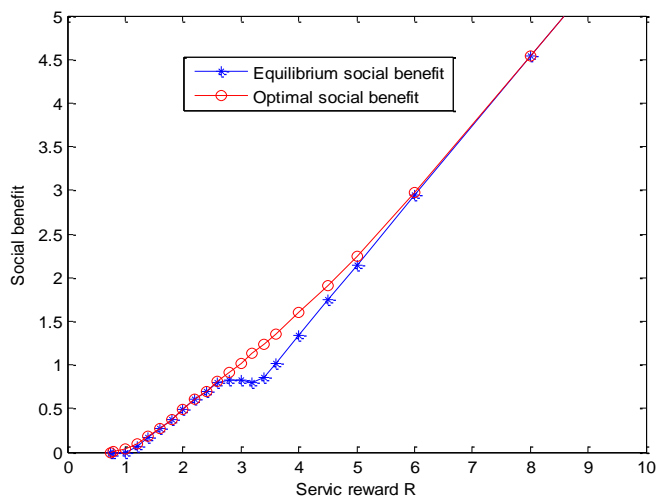

(b)

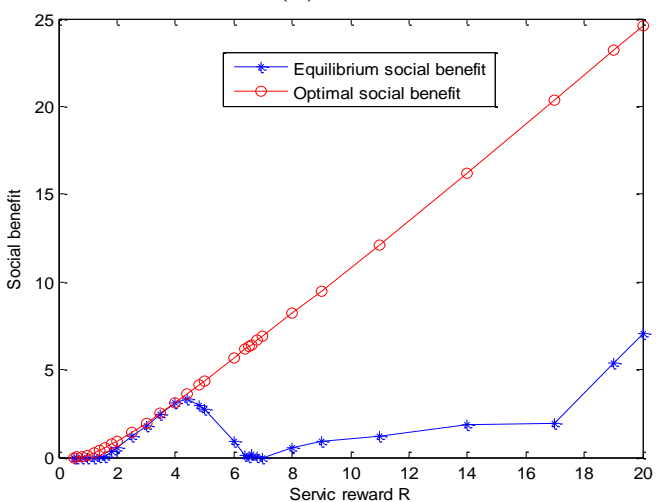

Figure 3. Equilibrium and Optimal Social Benefit of SUs with Respect to $R$, a for $\lambda=0.8, \mu=2, \theta=0.3, \eta=0.6, C=1$; b for $\lambda=1.7, \mu=2.4, \theta=0.1, \eta=0.3$,

$$
C=1
$$

\section{Conclusion}

In this paper we investigate the admission control mechanism for SUs from the economic viewpoint based on partially observable $\mathrm{M} / \mathrm{M} / 1$ queueing theory. We propose two different access strategies to maximize the net benefit of individual SU and overall society respectively. There are still many research issues that can be extended from our proposed model. In the future, we could explore the decision making procedure of SUs by applying M/G/1 queueing theory.

\section{References}

[1] C. W. Wang, L. C. Wang and F. Adachi, "Modeling and analysis of multiuser spectrum selection schemes in cognitive radio networks", Proceedings of IEEE International Symposium on PIMRC, , Tokyo, Japan, (2009) September, pp. 828-832.

[2] X. Li, J. Wang, H. Li and S. Q. Li, "Delay analysis and optimal access strategy in multichannel dynamic spectrum access system", 2012 International Conference on Computing, Networking and Communication, Hawaii, USA, (2012) February, pp. 376-380.

[3] H. S. Li and Z. Han, "Socially optimal queuing control in cognitive radio systems: pricing and learning", 2010 IEEE Wireless Communications and Networking Conference, Sydney, Australia, (2010) April 1-6.

[4] C. T. Do, N. H. Tran, M. V. Nguyen, C. S. Hong and S. Lee, "Social optimization strategy in unobserved queueing systems in cognitive radio networks", IEEE Communications Letters, vol. 16, (2012), pp. 1944-1947.

[5] R. Hassin and and M. Haviv, "To queue or not to queue: Equilibrium behavior in queueing systems", Kluwer Academic Publisher, Norwell, (2003). 
[6] I. F. Akyildiz, W. Y. Lee, M. C. Vuran and S. Mohanty, "NeXt generation/dynamic spectrum access/cognitive radio wireless networks: A survey, Computer Networks, vol. 50, (2006), pp. 2127 2159.

[7] A. Economou and S. Kanta, "Equilibrium balking strategies in the observable single-server queue with breakdowns and repairs", Operations Research Letters, vol. 36, (2008), pp. 696-699. 
International Journal of $u-$ and e- Service, Science and Technology Vol.7, No.2 (2014) 